\title{
Operative Vaginal Delivery and Invasive Procedures in Pregnancy among Women living with HIV
}

Helen Peters ${ }^{1}$ Kate Francis ${ }^{1}$, Kate Harding ${ }^{2}$, Pat A Tookey ${ }^{1}$, Claire Thorne ${ }^{1}$

${ }^{1}$ Population, Policy and Practice Programme, UCL GOS Institute of Child Health, University College London, London, United Kingdom

${ }^{2}$ Guys \& St Thomas' NHS Foundation Trust, London, United Kingdom

Corresponding Author: Helen Peters, Population, Policy, and Practice Programme, UCL GOS Institute of Child Health, 30 Guilford Street, London WC1N 1EH, United Kingdom

Email: helen.peters@ucl.ac.uk,Tel: +44 2079052693

Alternative contact: Dr Claire Thorne, Population, Policy, and Practice Programme, UCL GOS Institute of Child Health, 30 Guilford Street, London WC1N 1EH, United Kingdom

Email: claire.thorne@ucl.ac.uk, Tel: +44 2079052105

\section{Abstract}

Objectives To describe the use and outcomes of operative delivery and invasive procedures in pregnancy amongst women living with HIV.

Study Design The National Study of HIV in Pregnancy and Childhood (NSHPC) is a comprehensive population-based surveillance study in the UK and Ireland. The NSHPC has collected data on operative delivery since 2008, and invasive procedures in pregnancy (amniocentesis, cordocentesis, chorionic villus sampling) from 2012. Descriptive analyses were conducted on 278 pregnancies expected to deliver from 1 January 2008 with outcome reported to the NSHPC by 31 March 2016.

Results Among 9372 pregnancies in 2008-2016, there were 9072 livebirths with 251 operative deliveries and 27 invasive procedures in pregnancy reported.

Information was available for $3023 / 3490$ vaginal deliveries, and use of forceps or vacuum reported in with 249 deliveries (8.2\%), increasing over calendar time to almost 10\% by 2014-16. Forceps were 
used twice as often as vacuum delivery, and forceps use increased over time. One infant delivered operatively is known to have acquired HIV.

From 2012 there were 4063 pregnancies resulting in 3952 livebirths, 83 terminations and 28 stillbirths. 2163/4063 had information on use (or not) of invasive procedures in pregnancy. Amniocentesis was reported in 25/2163 pregnancies, there was one report of chorionic villus sampling and one of cordocentesis. There were no reported transmissions following invasive procedures in pregnancy.

\section{Conclusions}

This is the largest study to date to report on operative delivery in women living with HIV on CART, and provides an up-to-date picture of invasive procedures during pregnancy in this group. Findings from this comprehensive national study are reassuring but numbers are currently low; on-going monitoring is crucial as obstetric care of women with HIV becomes normalised.

Keywords: HIV; pregnancy; operative vaginal delivery; amniocentesis; invasive procedures 


\section{Introduction}

A high and increasing proportion of pregnant women living with HIV are achieving viral suppression by the time of delivery (in the UK this was $80 \%$ in 2007-2011[1] and $90 \%$ in 2012-2014[2] . The UK continues to move towards ensuring that these women have the same obstetric options as their uninfected peers, with such "normalising" of obstetric care remaining a priority both in guidelines and practice. A key example of this is the recommendation for vaginal delivery in this group $[3,4]$.

As planned vaginal deliveries increase, the number of women requiring operative vaginal deliveries is also likely to rise. Historically, operative delivery has been avoided in women living with HIV owing to theoretical fears of mother-to-child transmission (MTCT)[3]. Although studies from the pre-combined antiretroviral therapy (CART) era suggested little or no increase in MTCT risk associated with operative vaginal deliveries $[5,6]$ these were based on small numbers. The 2008 BHIVA guidelines [7] recommended avoidance of mid or rotational forceps and favoured low cavity forceps over vacuum delivery if operative vaginal delivery was necessary; since 2012 guidance has been revised to indicate that if $V L$ is suppressed the most appropriate instrument should be used, consistent with national obstetric guidelines[3]. Contemporary data on operative delivery in women living with HIV are lacking, with studies in the cART era not re-examining these factors[3].

Invasive procedures in pregnancy were traditionally avoided where possible in women living with HIV because research before the pre-CART era suggested they were associated with an increase in MTCT rates [5,8,9]. With improved methods of trisomy-21 screening and use of non-invasive prenatal testing (NIPT), the need for invasive antenatal testing has (and continues to) decline in the UK, although on some occasions amniocentesis is still needed for optimum management. World literature on use of invasive procedures in the CART era has been sparse [9-11], and although the small numbers have precluded firm conclusions, it has been suggested that there is no increased risk of transmission. The most recent study from Italy presents data since 2001 and reinforces these findings [9]. Current British HIV Association (BHIVA) guidelines [3] recommend combined screening (including nuchal translucency testing) to reduce the number of invasive investigations; where required the procedure should be deferred until the viral load $(V L)$ is suppressed if possible.

Our aim is to present the current picture regarding operative deliveries and the use of invasive procedures in pregnancy among women living with HIV in the UK and Ireland, using data from the National Study of HIV in Pregnancy and Childhood (NSHPC).

\section{Materials and Methods}

The NSHPC is a well-established comprehensive, active surveillance study collecting data on all pregnancies and deliveries in diagnosed women living with HIV in the UK and Ireland since 1990. HIV-exposed infants are followed up through paediatricians to establish infection status. To date details on over 20,000 pregnancies are held by the Study. Full details are described elsewhere $[12,13]$. 
Details about operative delivery have been routinely requested by the NSHPC since 2008. Information regarding invasive procedures in pregnancy (amniocentesis, cordocentesis andchorionic villus sampling (CVS)) have been requested since 2012.

Analyses were based on pregnancies to diagnosed women with known outcomes, excluding miscarriages, reported between January 2008 and the end of March 2016:

Analysis 1: operative deliveries: 3663 pregnancies with expected date of delivery (EDD) from $1^{\text {st }}$ January 2008 onwards, resulting in a vaginally delivered livebirth. Information on use of instruments was available for $3023 / 3663$ deliveries (82.6\%) (Figure 1). Characteristics in pregnancy are reported per pregnancy, whereas outcome and delivery characteristics are reported per infant (as we include multiple births).

Analysis 2: invasive investigations during pregnancy: 4063 pregnancies with EDD from $1^{\text {st }}$ January 2012 onwards. Information was available for 2163/4063 pregnancies (53.2\%) (Figure 2).

Maternal VL at the time of delivery and/or the invasive antenatal procedure was categorised as undetectable $(<50$ copies $/ \mathrm{ml})$ or detectable $(\geq 50)$.

Infant's HIV infection status was classified as uninfected if a negative polymerase chain reaction (PCR) test was reported after one month of age or a negative antibody test after 18 months of age, and infected if a positive PCR was reported at any time or a positive antibody test reported after 18 months of age. Some infants were below the age at which infection status could be confirmed ( 18 month antibody test), and so were excluded where appropriate.

Data analysis was mostly descriptive owing to the very small numbers of invasive procedures and operative deliveries. Where a vaginal delivery occurred, characteristics of operative and non-operative deliveries were compared. Categorical variables were compared using $\chi^{2}$ tests or Fisher's exact tests.

Data were managed in Access 2010, and analysed using Stata version 13.1 (StataCorp LP, College Station, USA). 


\section{Results}

There were 9372 pregnancies (in 7417 women) with an EDD in 2008-2016, with 4063 pregnancies (3952 deliveries) from 2012 onwards. Overall, 9072 (96.8\%) were live deliveries, $217(2.3 \%)$ terminations and $83(0.9 \%)$ stillbirths. Among the deliveries resulting in a livebirth, 3663 (40.4\%) were vaginal, 3054 (33.7\%) elective caesarean sections and 2331 (25.7\%) emergency caesarean sections ( 24 missing mode of delivery). There were 211 twin and three triplet deliveries.

\section{Operative delivery}

Of the 3023 vaginal deliveries with data, 249 (8.2\%) were reported as operative (Figure 1); seven were twin deliveries (in three, operative delivery was for one twin only), giving 251 babies delivered by forceps or vacuum. Median age at delivery was 33 years (IQR: 28, 36), with no difference in age according to operative delivery (Table 1). Women having an operative delivery were more likely to be in their first pregnancy $(p<0.001)$, and delivering at term $(p=0.02)$.

Forceps were used more than twice as often as vacuum (Table 1). Both forceps and vacuum were used in three deliveries. Type of forceps was reported in around half of cases (82/169), with non-rotational forceps reported in the majority (76/82).

The proportion of operative vaginal deliveries increased over time from $7.3 \%$ (75/1032) in 2008-10 to 8.3\% (107/1284) in 2011-13 and 9.5\% (67/707) in 2014-16, but the increase was non-significant $(p=0.13)$. There was some evidence of a change of type of operative delivery over time: in 2008-10 4.0\% (41/1032) of vaginal births were delivered with forceps compared with $7.4 \%(52 / 707)$ in $2014-16(p<0.01)$, whereas the proportion of vacuum deliveries remained fairly stable over time, at $2.7 \%(28 / 1032)$ in $2008-10$ versus $2.1 \%$ $(15 / 707)$ in 2014-16 ( $p=0.55)$.

Over two-fifths of operative delivery reports (107/249) were from London, in keeping with the proportion of total deliveries reported from the London region (43\%).

Of the 251 infants, 29 did not have a confirmed infection status, of whom 18 had not reached 18 months at the time of the analysis. Excluding these 18 infants, infection status had been confirmed for $95 \%(222 / 233)$ of infants. One infant was reported to be infected, but there were other significant risk factors (including maternal adherence issues and possible breastfeeding) that could have contributed to this transmission. For this case there were insufficient data to determine whether the transmission occurred intrapartum or not

\section{Invasive Investigations in Pregnancy}

From January 2012, amniocentesis was reported in 25 pregnancies, and CVS and cordocentesis in one pregnancy each. Median maternal age among women having invasive investigations was 38 years (IQR: 33, 41 years), compared with 33 years (IQR: 29, 27 years) for all pregnancies reported in 2012-2016. The 27 reports were from 23 different units, 14 
of which were in London. Amniocentesis was conducted at a median of 17 weeks gestation (range: 13, 25 weeks); no woman had more than one procedure. The single CVS procedure was carried out at 11 weeks gestation. Of the 27 women with an invasive investigation, 26 were on CART at the time of the procedure: 25 had undetectable VL and one woman had a $\mathrm{VL}$ of 200 copies/ml after 10 days on CART. The one woman not on CART had an undetectable $V L$ at the time of amniocentesis (and also received intravenous zidovudine during the procedure). Cordocentesis was carried out several times during the one pregnancy reported with this procedure, as part of the management of fetal haemolytic disease; maternal VL was undetectable on each occasion.

The 27 pregnancies ended in 23 livebirths (all singletons), two stillbirths and two terminations of pregnancy. Both terminations and one of the still births followed the detection of chromosomal abnormalities, with one further chromosomal abnormality (trisomy-18) reported in infant pregnancy which resulted in a live born infant (and subsequent neonatal death). One additional live born infant had spina bifida. Of the 22 surviving infants, 20 were aged $>18$ months at the time of analysis, with all 20 having infection status confirmed; no HIV infections have been reported to date.

\section{Comment}

This is the largest study to date reporting on operative vaginal delivery in women living with HIV, and rates of such deliveries are increasing whilst improved non-invasive screening methods may reduce the need for invasive investigations during pregnancy. Although the number of pregnancies involving such procedures remains small, this national surveillance study provides a comprehensive picture and evidence to support previous literature and guidelines recommending the "normalisation" of delivery amongst HIV positive women $[5,14-16]$.

Reflecting the high proportion of women now approaching delivery with an undetectable VL, the overall vaginal delivery rate in the NSHPC increased from $32 \%$ in 2009 to $50 \%$ in 2014 (results not presented). Operative delivery was reported in one in 12 vaginal deliveries occurring in 2008 onwards, increasing over calendar time to almost one in 10 by 2014 to 2016. Over this period there was an increase in the proportion of forceps deliveries amongst women living with HIV, whereas the proportion of vacuum deliveries remained fairly stable, perhaps reflecting previous guidance for obstetricians to use the instrument perceived to cause the least neonatal skin trauma [7].

Since the start of 2012, about $1 \%$ of pregnancies in women living with HIV were reported to have had an invasive antenatal procedure, with amniocentesis predominating. In all but one case, women had an undetectable $\mathrm{VL}$ at the time of the procedure (as recommended by 2012 BHIVA guidelines [3]); the exception was a woman with low and declining VL prior to the procedure. Women with HIV are slightly more likely to have a false positive Downs screening result than other women [17], potentially resulting in increased rates of invasive prenatal investigations. The National Screening Committee recently proposed that NonInvasive Prenatal Testing (NIPT) be offered to women deemed to be high risk following the 
current primary screen [18]. Although an invasive procedure will still be required for diagnostic purposes, the introduction of NIPT may reduce the number of invasive procedures overall.

To date among the 251 pregnancies with operative delivery and the 27 pregnancies with invasive procedures in pregnancy reported, one vertical transmission has been confirmed in the group with operative delivery. It is important to note that there were other complicating factors contributing to this case, and there were insufficient data to determine whether the transmission occurred intrapartum or not. Our findings are thus consistent with current overall MTCT rates [1] in this population, which are around 5 per 1000. Overall, infection status was available for $95 \%$ of infants over 18 months, with missing data largely due to recently-born infants awaiting infection status confirmation and not likely to result in substantial bias. We have previously shown that cases reported at a later date did not affect transmission rates [1].

The current national rate of operative deliveries amongst vaginal deliveries in England is $17.7 \%$ [19], substantially higher than the $8.2 \%$ in our study. Nationally, forceps and vacuum delivery account for $9.6 \%$ and $8.1 \%$ of vaginal deliveries respectively [19] in contrast to $5.6 \%$ and 2.4\% amongst our population. According to 2010 RCOG Green Top Guidelines[20] , amniocentesis was offered to $5 \%$ of the UK general antenatal population. Although the overall rate of prenatal invasive testing has recently reduced in the UK (owing to improved screening and NIPT availability), current rates of uptake of NIPT in women living with HIV are unclear it may be higher than the $1 \%$ reported here.

Although national figures can provide the context for current practice, the complex choices made throughout the management of the pregnancies of women living with HIV mean that it is not appropriate to directly compare this group with the general population. Our surveillance study does not collect sufficiently detailed data to define the myriad factors affecting clinical decision-making during pregnancy and delivery. A woman's HIV status may be only one of several considerations in a process focused on optimising the health of both mother and baby, with a range of factors driving the choices surrounding timing and type of procedure. This is illustrated by the cordocentesis case here, where repeated procedures were required for appropriate management of fetal haemolytic disease.

Maternal age has been increasing amongst the HIV-population [21], reflecting increased survival rates as well as the general trend amongst the obstetric population [22]. One in ten deliveries in the NSHPC are now in women aged 40 years or older, and this has implications for pregnancy management (for example, the increased risk of chromosomal abnormalities) making this a growing area for research.

A limitation of this analysis was the small number of invasive procedures in pregnancy (23 liveborn infants), historically avoided in management of HIV-positive pregnancies, meaning that our analysis was solely descriptive. Although the NSHPC does seek pregnancy outcome details where miscarriages and terminations occur, there is known to be under-reporting in this area. The number of cases of FBS and fetal scalp monitoring available in the NSHPC was too small to offer any insight, and data on episiotomy and perineal status are not collected 
by the NSHPC. A larger number of operative deliveries (251) provided opportunity for more detailed investigation; however details provided regarding type of forceps were missing in many cases. .

Our findings should reassure those involved with managing pregnancy and delivery in women living with HIV, who need to balance concerns around vertical transmission risk (very low in women on CART with suppressed VL) with the need for appropriate obstetric management and advice for women. The ageing population of pregnant women with HIV, alongside new antenatal screening practices (e.g. NIPT) underscore the need to continue monitoring frequency and outcomes of invasive antenatal investigations. However, the increasing vaginal, and operative, deliveries reflecting continued treatment success and normalisation of obstetric care, are of key interest and require continued surveillance in order to maintain confidence in the reassuring outcomes presented here.

\section{Acknowledgements:}

National surveillance of obstetric and paediatric HIV is undertaken through the National Study of HIV in Pregnancy and Childhood (NSHPC) in collaboration with the Health Protection Agency Centre for Infections, now Public Health England, and Health Protection Scotland. The authors gratefully acknowledge the contribution of the midwives, obstetricians, genitourinary physicians, paediatricians, clinical nurse specialists, and all other colleagues who report to the NSHPC through the British Paediatric Surveillance Unit of the Royal College of Paediatrics and Child Health, and the obstetric reporting scheme run under the auspices of the Royal College of Obstetricians and Gynaecologists. We also thank Rebecca Sconza (Research Assistant), Anna Horn (Study Assistant) and Icina Shakes (former Study Assistant) for their essential contributions to the NSHPC.

The National Study of HIV in Pregnancy and Childhood receives core funding from Public Health England, with additional funding from the Public Health England National Health Service Infectious Diseases in Pregnancy Screening Programme. The UCL Institute of Child Health receives a proportion of funding from the Department of Health's National Institute for Health Research Biomedical Research Centres funding scheme. 


\section{References}

1. Townsend $\mathrm{CL}$, Byrne L, Cortina-Borja $\mathrm{M}$, et al. Earlier initiation of ART and further decline in mother-to-child HIV transmission rates, 2000-2011. AIDS Lond Engl. 2014 Apr 24;28(7):1049-57.

2. Peters $\mathrm{H}$, Francis $\mathrm{K}$, Sconza R, et al. UK mother-to-child transmission rates continue to decline: 2012-2014. Clin Infect Dis. 2016 (in Press)

3. BHIVA-Pregnancy-guidelines-update-2014.pdf [Internet]. [cited 2015 Nov 26]. Available from: http://www.bhiva.org/documents/Guidelines/Pregnancy/2012/BHIVA-Pregnancy-guidelinesupdate-2014.pdf

4. Aebi-Popp K, Mulcahy F, Rudin C, et al. National Guidelines for the prevention of mother-tochild transmission of HIV across Europe - how do countries differ? Eur J Public Health. 2013 Dec 1;23(6):1053-8.

5. Mandelbrot L, Mayaux MJ, Bongain A, et al. Obstetric factors and mother-to-child transmission of human immunodeficiency virus type 1: the French perinatal cohorts. SEROGEST French Pediatric HIV Infection Study Group. Am J Obstet Gynecol. 1996 Sep;175(3 Pt 1):661-7.

6. Kind C, Rudin C, Siegrist CA, et al. Prevention of vertical HIV transmission: additive protective effect of elective Cesarean section and zidovudine prophylaxis. Swiss Neonatal HIV Study Group. AIDS Lond Engl. 1998 Jan 22;12(2):205-10.

7. British HIV Association and Children's HIV Association guidelines for the management of HIV infection in pregnant women 2008 [Internet]. [cited 2015 Nov 26]. Available from: http://www.bhiva.org/documents/Guidelines/Pregnancy/2008/PregnancyPub.pdf

8. Maiques V, García-Tejedor A, Perales A, Navarro C. Intrapartum fetal invasive procedures and perinatal transmission of HIV. Eur J Obstet Gynecol Reprod Biol. 1999 Nov;87(1):63-7.

9. Maiques V, García-Tejedor A, Perales A, Córdoba J, Esteban RJ. HIV detection in amniotic fluid samples. Amniocentesis can be performed in HIV pregnant women? Eur J Obstet Gynecol Reprod Biol. 2003 Jun 10;108(2):137-41.

10. Coll O, Suy A, Hernandez S, et al. Prenatal diagnosis in human immunodeficiency virusinfected women: a new screening program for chromosomal anomalies. Am J Obstet Gynecol. 2006 Jan;194(1):192-8.

11. Somigliana E, Bucceri AM, Tibaldi C, et al. Early invasive diagnostic techniques in pregnant women who are infected with the HIV: a multicenter case series. Am J Obstet Gynecol. 2005 Aug;193(2):437-42.

12. Townsend $\mathrm{CL}$, Cortina-Borja $\mathrm{M}$, Peckham CS, et al. Low rates of mother-to-child transmission of HIV following effective pregnancy interventions in the United Kingdom and Ireland, 2000-2006. AIDS Lond Engl. 2008 May 11;22(8):973-81.

13. Townsend CL, Cortina-Borja M, Peckham CS, Tookey PA. Trends in management and outcome of pregnancies in HIV-infected women in the UK and Ireland, 1990-2006. BJOG Int J Obstet Gynaecol. 2008 Aug;115(9):1078-86.

14. L. Mandelbrot, C. Jasseron, ANRS French Perinatal Cohort. Amniocentesis and mother-tochild human immunodeficiency virus transmission in the Agence Nationale de Recherches sur le SIDA et les Hépatites Virales French Perinatal Cohort. In Boston MA; 2008. 
15. Ekoukou D, Khuong-Josses M-A, Ghibaudo N, Mechali D, Rotten D. Amniocentesis in pregnant HIV-infected patients. Eur J Obstet Gynecol Reprod Biol. 2008 Oct;140(2):212-7.

16. Simoes $M$, Marques $C$, Albergaria $F$, et al. Amniocentesis is a low-risk procedure in HIVtreated pregnant women. J Int AIDS Soc. 2010;13(Suppl 4):P163.

17. Charlton TG, Franklin JM, Douglas M, et al. The impact of HIV infection and antiretroviral therapy on the predicted risk of Down syndrome. Prenat Diagn. 2014 Feb;34(2):121-7.

18. Current UK NSC recommendations [Internet]. [cited 2016 Jun 10]. Available from: http://legacy.screening.nhs.uk/screening-recommendations.php

19. Find data - Health \& Social Care Information Centre [Internet]. [cited 2015 Nov 26]. Available from:

http://www.hscic.gov.uk/searchcatalogue?q=maternity+statistics\&area=\&size=10\&sort=Relevance\# top

20. RCOG Green Top Guidelines 2010 [Internet]. [cited 2015 Nov 26]. Available from: https://www.rcog.org.uk/globalassets/documents/guidelines/gtg_8.pdf

21. Townsend, C, de Reuiter, A, Peters, H. et al. Pregnancies in older women living with HIV in the United Kingdom and Ireland HIV Med. doi:10.1111/hiv.12469

22. Office for National Statistics. Characteristics of Mother 1, England and Wales, 2013. 2014 [Internet]. Available from: http://www.ons.gov.uk/ons/rel/vsob1/characteristics-of-Mother-1-englandand- wales/2013/stb-characteristics-of-mother-1--2013.html 


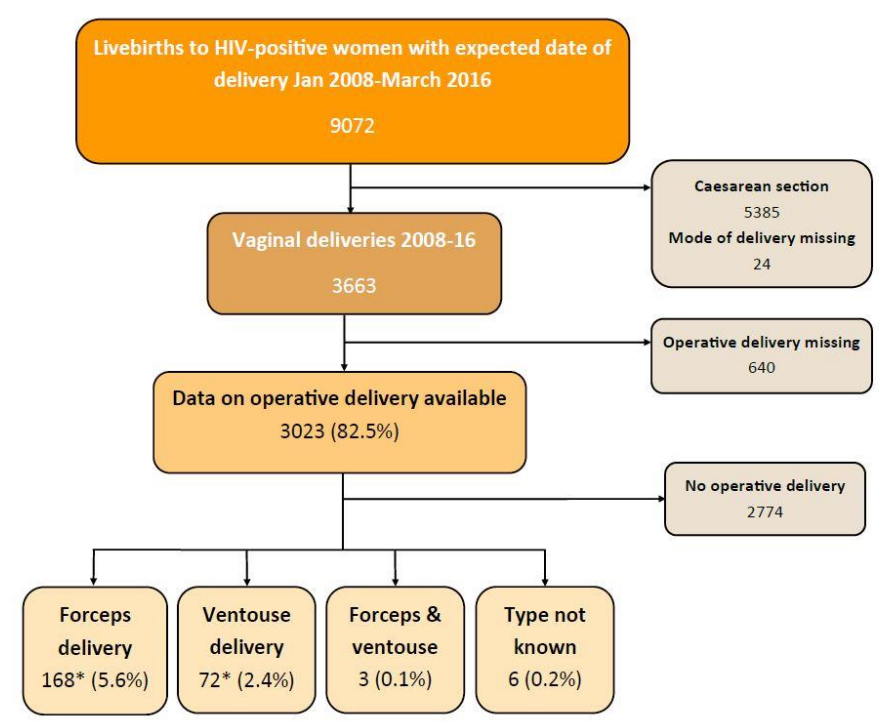

*169 infants had a forceps delivery (including one set of twins where both infants had a forceps delivery), 73 infants delivered by ventouse (including one set of twins where both infants had a ventouse delivery)

Figure 1: Flowchart of operative vaginal deliveries

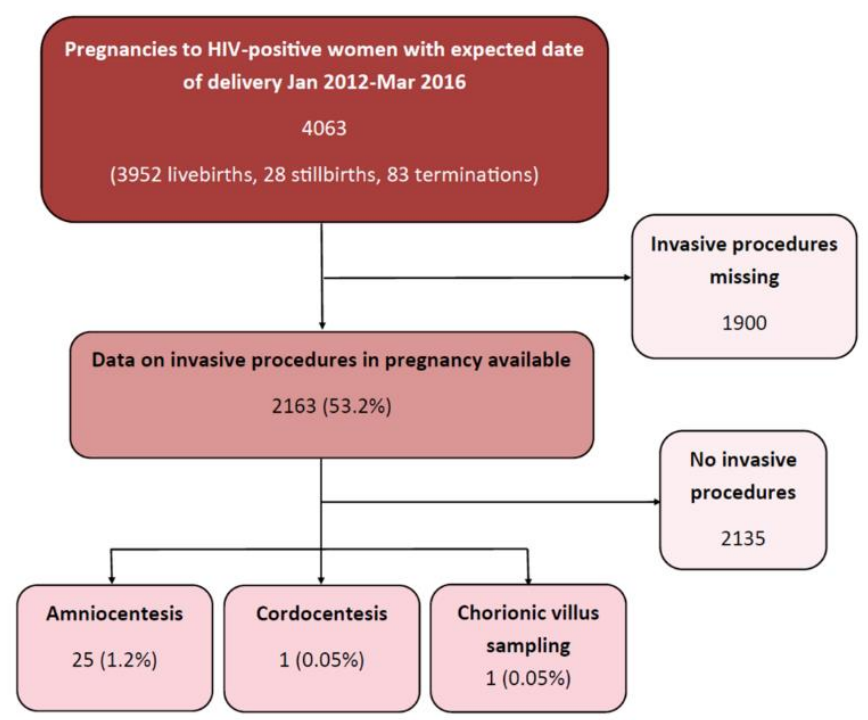

Figure 2: Flowchart pregnancies with invasive procedures 
Table 1: Characteristics of pregnancies resulting in a vaginal delivery, by operative delivery

\begin{tabular}{|c|c|c|c|}
\hline & $\begin{array}{l}\text { Operative } \\
\text { Deliveries }\end{array}$ & $\begin{array}{l}\text { No Operative } \\
\text { Delivery }\end{array}$ & $p$-value \\
\hline \multicolumn{4}{|l|}{ Maternal Age (years) } \\
\hline$<25$ & $26(10.4 \%)$ & 237 (8.5\%) & 0.27 \\
\hline $25-29$ & $65(26.1 \%)$ & $613(22.1 \%)$ & \\
\hline $30-34$ & $82(32.9 \%)$ & $962(34.7 \%)$ & \\
\hline$>34$ & $76(30.5 \%)$ & $962(34.7 \%)$ & \\
\hline \multicolumn{4}{|l|}{ Parity } \\
\hline 0 & 132 (55.4\%) & $555(20.9 \%)$ & $<0.001$ \\
\hline 1 & 76 (31.9\%) & $967(36.3 \%)$ & \\
\hline$\geq 2$ & $30(12.6 \%)$ & 1137 (42.8\%) & \\
\hline Missing & 11 & 115 & \\
\hline \multicolumn{4}{|l|}{ Birthweight (kg)* } \\
\hline$<2.5$ & $15(6.1 \%)$ & $248(9.0 \%)$ & 0.11 \\
\hline $2.5-4.5$ & 230 (93.1\%) & 2472 (90.5\%) & \\
\hline$>4.5$ & $1(0.4 \%)$ & $12(0.4 \%)$ & \\
\hline Missing & 5 & 64 & \\
\hline \multicolumn{4}{|l|}{$\begin{array}{l}\text { Gestational Age } \\
\text { (weeks)* }\end{array}$} \\
\hline$<37$ & $10(4.0 \%)$ & $223(8.0 \%)$ & 0.02 \\
\hline$\geq 37$ & 239 (96.0\%) & 2549 (92.0\%) & \\
\hline Missing & 2 & 24 & \\
\hline \multicolumn{4}{|l|}{ Instrument Type* } \\
\hline Forceps & 169 (67.3\%) & & \\
\hline - rotational/Keilland & 6 & & \\
\hline - non-rotational & 76 & & \\
\hline - type not specified & 87 & & \\
\hline Vacuum & 73 (29.0\%) & & \\
\hline Forceps \& Vacuum & $3(1.2 \%)$ & & \\
\hline Not known & 6 & & \\
\hline
\end{tabular}

* Outcome variables include twins deliveries ( $n=251$ for operative deliveries and 2796 for non-operative deliveries) 